\title{
Hopf Bifurcation Analysis in a New Chaotic System with Chaos Entanglement Function
}

\author{
Zhang Jiangang, Chu Yandong, Du Wenju, Chang Yingxiang, and An Xinlei \\ Department of Mathematics, Lanzhou Jiaotong University, Lanzhou 730070, China \\ Correspondence should be addressed to Chu Yandong; shuliky0931@126.com
}

Received 28 March 2014; Revised 30 June 2014; Accepted 30 June 2014; Published 22 July 2014

Academic Editor: Maoan Han

Copyright (C) 2014 Zhang Jiangang et al. This is an open access article distributed under the Creative Commons Attribution License, which permits unrestricted use, distribution, and reproduction in any medium, provided the original work is properly cited.

\begin{abstract}
A new approach to generate chaotic phenomenon, called chaos entanglement, is introduced in this paper. The basic principle is to entangle two or multiple stable linear subsystems by entanglement functions to form an artificial chaotic system such that each of them evolves in a chaotic manner. The Hopf bifurcation of a new chaotic system with chaos entanglement function is studied. More precisely, we study the stability and bifurcations of equilibrium in the new chaotic system. Besides, we controlled the system to any fixed point to eliminate the chaotic vibration by means of sliding mode method. And the numerical simulations were presented to confirm the effectiveness of the controller.
\end{abstract}

\section{Introduction}

The discovery of the eminent Lorenz system [1] has led to an extensive study of chaotic behaviors in nonlinear systems due to many possible applications in science and technology. In the last three decades, many new three-dimension chaotic systems have been proposed, such as Rossler system [2], Chen and Ueta system [3], Lü and Chen system [4], Liu et al. system [5], and generalized Lorenz system family [6]. Later, many Lorenz-like or Lorenz-based chaotic systems were proposed and investigated. Some classical 3D autonomous chaotic systems have three particular fixed points: one saddle and two unstable saddle-foci [7]. The other 3D chaotic systems have two unstable saddle-foci $[8,9]$. Yang and Chen [10] found another 3D chaotic system with three fixed points: one saddle and two stable equilibriums. Recently, Qi et al. [11] constructed a new three-dimensional chaotic system which has complex dynamics with some interesting characteristics. Yang and Chen [12] analyzed the complex dynamics of the unified Lorenz-type system with six parameters, which contain common chaotic systems as its particular cases.

As a representative of the nonlinear robust control theory, variable structure control theory has been widely researched throughout the world and also had an increasing number of industrial applications. Chen et al. [13] studied the nonlinear dynamics behavior of hydroturbine governing system. In order to eliminate the chaotic vibration, the author used sliding mode method and controlled the system to any fixed point and any periodic orbit. The results show that using sliding mode method can make the system track target orbit strictly and smoothly with short transition time. Chen et al. [14] proposed a no-chattering sliding mode control strategy for a class of fractional-order chaotic systems. The designed control scheme guarantees the asymptotical stability of an uncertain fractional-order chaotic system. Lee et al. [15] present a sliding mode controller with integral compensation for a magnetic suspension balance beam system. The control scheme comprises an integral controller which is designed for achieving zero steady-state error under step disturbances and a sliding mode controller which is designed for enhancing robustness under plant uncertainties. Wang et al. [16] present two methods to design a single-input/single-output integral variable structure system. To ensure the robustness of the system control, Chen et al. [17] stabilized the chaotic orbits to arbitrary chosen fixed points and periodic orbits by means of sliding mode method, and Matlab simulations were presented to confirm the validity of the controller.

Over the past one decade, as we have seen, researchers have paid great attention to generate chaos and analyze its dynamics, because more and more chaotic phenomena 
have been found in many research fields, and chaos has powerful applications in secure communication, information processing, nonlinear circuits, biological systems, chemical reactions, and so forth. Thus, the requirement for creating new chaotic systems is dramatically increasing. A new approach to generate chaotic phenomenon, called chaos entanglement, is introduced in this paper. The basic principle is to entangle two or multiple stable linear subsystems by entanglement functions to form an artificial chaotic system such that each of them evolves in a chaotic manner. In this paper, we mainly consider a new chaotic system constructed by Zhang et al. [18]. Zhang proposed a new approach to generate chaotic phenomenon, called chaos entanglement. And he constructed a three-dimensional continuous autonomous chaotic system. However, the basic dynamical properties of the system and the relationship between the Hopf bifurcation and the system parameters have not been clarified yet.

This paper is organized as follows. We first described the new chaotic system in Section 2. In Section 3 we present the linear analysis of equilibriums of system (1). A brief review of the methods used to study codimension one Hopf bifurcations is presented in Section 4. In Section 5, numerical simulation is given to illustrate the theoretical analysis. We controlled the system to any fixed point to eliminate the chaotic vibration by means of sliding mode method in Section 6. Finally, conclusions are given in Section 7.

\section{New Chaotic Attractor}

Chaos Entanglement [18]. If two or more linear subsystems can behave in chaotic manners when they are entangled by some nonlinear functions, we say it is chaos entanglement. These nonlinear functions are called entanglement functions.

Consider two linear subsystems; one is 2-dimensional and the other is 1-dimensional,

$$
\begin{gathered}
\dot{x}=a x+b y, \\
\dot{y}=-b x+a y, \\
\dot{z}=c z,
\end{gathered}
$$

where $(x, y, z) \in R^{3}$ is the state variable. When $a<0$ and $c<0$, both subsystems are stable. We entangle these two subsystems in the following way:

$$
\begin{gathered}
\dot{x}=a x+b y, \\
\dot{y}=-b x+a y+d \sin z, \\
\dot{z}=c z+e \sin x,
\end{gathered}
$$

where $a<0, c<0,(d, e) \in R^{2}$ is the entanglement coefficient, and $(\sin x, \sin z)$ is the entanglement function.

Zhang et al. [18] have proved that the system (3) is dissipative and bounded and possesses one positive Lyapunov exponent. So, the system (3) is chaotic. Despite the simplicity, system (3) has a rich dynamical behavior, ranging from stable equilibrium points to periodic and even chaotic oscillations, depending on the parameter values. When the real constants $a=-1, b=1, c=-1, d=36$, and $e=18$, the system (3) has a chaotic attractor as shown in Figures $1(\mathrm{a})-1(\mathrm{~d})$.

Moreover, the dynamics of the system (3) can be characterized with its Lyapunov exponents which are computed numerically by Wolf et al. algorithm proposed in [19], where the Lyapunov exponents $\lambda_{1}=0.596592, \lambda_{2}=$ 0 , and $\lambda_{3}=-3.596124$ as shown in Figure $2(a)$, and the Lyapunov dimension $D_{K Y}=2.16589$. The time history, the frequency spectrum, and Poincaré map in $y-z\{x=0\}$ plane of the chaotic attractor shown in Figures 2(b), 2(c) and 2(d) respectively.

\section{Linear Analysis of the New Chaotic System}

This paper studies the stability and bifurcations with the respective qualitative changes in the dynamics of the system (3). System (3) has a rich dynamical behavior, ranging from stable equilibrium points to periodic and even chaotic oscillations, depending on the parameter values.

In a vectorial notation which will be useful in the calculations, system (3) can be written as $x^{\prime}=(x, \zeta)$, where

$$
f(x, \zeta)=(a x+b y,-b x+a y+d \sin z, c z+e \sin x),
$$

$x=(x, y, z) \in R^{3}, \zeta=(a, b, c, d, e) \in R^{2}$.

The equilibria of system (3) can be found by solving the following equations simultaneously:

$$
\begin{gathered}
a x+b y=0, \\
-b x+a y+d \sin z=0, \\
c z+e \sin x=0 .
\end{gathered}
$$

Usually, it is not easy to find out its accurate solutions. For simplification, we only consider $E_{0}=(0,0,0)$.

For the sake of completeness we state the following lemma (Routh-Hurwitz stability criterion) whose proof can be found in $[6]$.

Lemma 1 (see [9]). The polynomial $p(\lambda)=\lambda^{3}+p_{1} \lambda^{2}+p_{2} \lambda+p_{3}$ with real coefficients has all roots with negative real parts if and only if the numbers $p_{1}, p_{2}, p_{3}$ are positive and the inequality $p_{1} p_{2}>p_{3}$ is satisfied.

We have the following proposition.

Proposition 2. Define the following subsets of $W$ :

$$
\begin{aligned}
& w_{1}=\{(a, b, c, d, e): \\
& \quad a<0, b>0, c<0, d>\frac{\left(2 a^{3}+2 a b^{2}+4 a^{2} c+2 a c^{2}\right)}{b e}, \\
& \quad e>0\},
\end{aligned}
$$




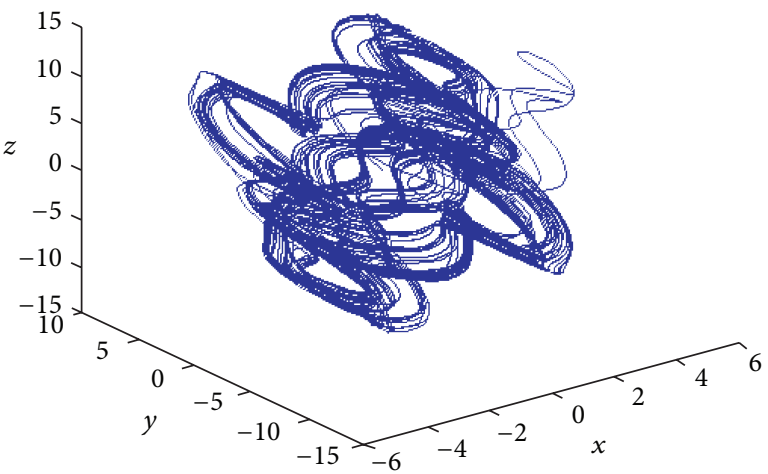

(a)

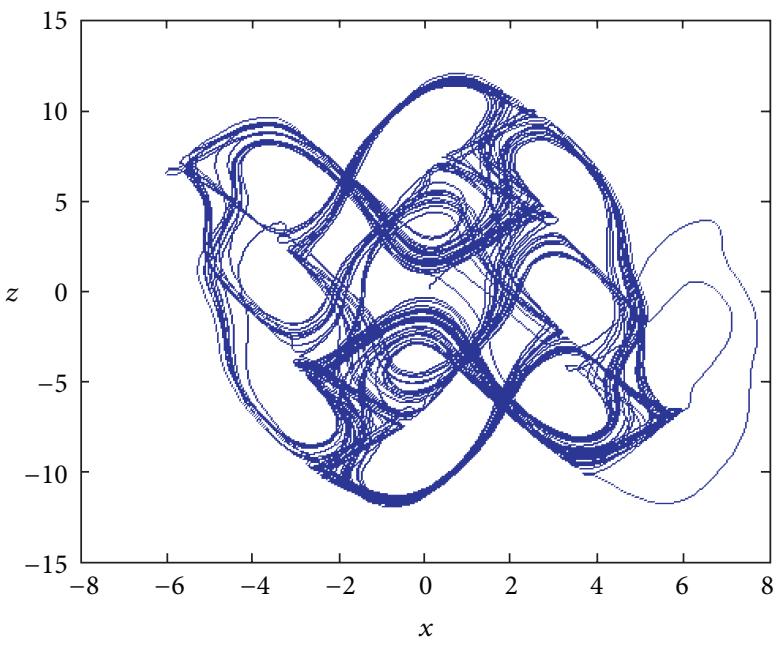

(c)

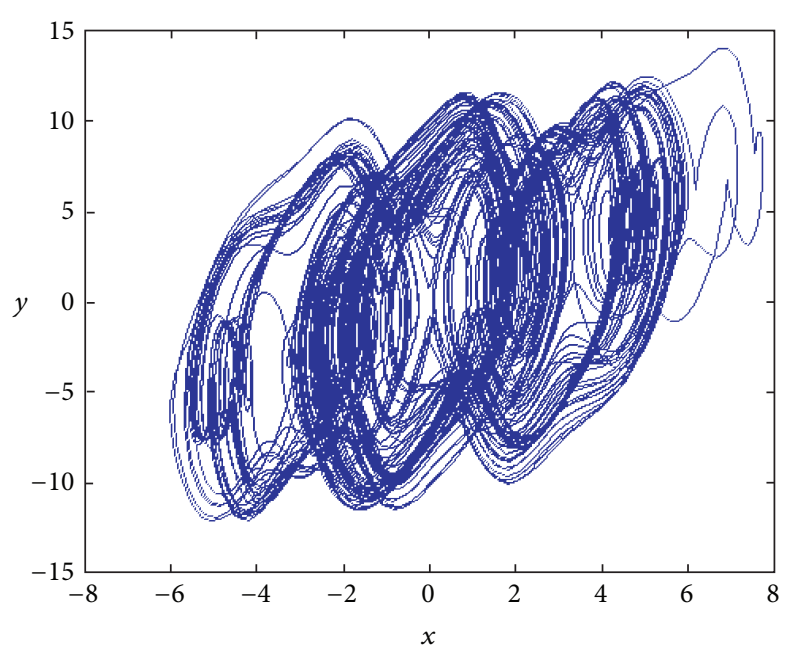

(b)

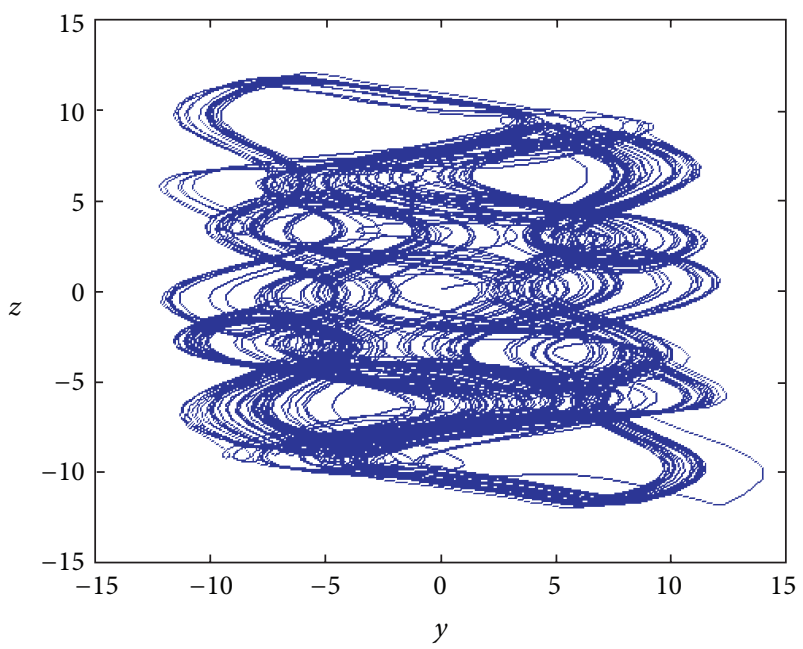

(d)

FIGURE 1: (a) Chaotic attractor given by (1) with $a=-1, b=1, c=-1, d=36$, and $e=18$, and projection of the chaotic attractor on the (b) $x-y$ plane, (c) $y-z$ plane, and (d) $x-z$ plane.

$$
\begin{aligned}
& w_{2}=\{(a, b, c, d, e): \\
& a<0, b>0, c<0, d<\frac{\left(2 a^{3}+2 a b^{2}+4 a^{2} c+2 a c^{2}\right)}{b e}, \\
& \quad e>0\}, \\
& w_{3}=\{(a, b, c, d, e): a<0, b>0, c<0, \\
& \left.\quad d=\frac{\left(2 a^{3}+2 a b^{2}+4 a^{2} c+2 a c^{2}\right)}{b e}, e>0\right\} .
\end{aligned}
$$

The following statements hold.
(1) If $(a, b, c, d, e) \in w_{1}$ then the equilibrium $E_{0}$ is locally asymptotically stable.

(2) If $(a, b, c, d, e) \in w_{2}$ then the equilibrium $E_{0}$ is unstable.

(3) If $(a, b, c, d, e) \in w_{3}$ then the equilibrium $E_{0}$ is unstable.

Proof. At the fixed point $E_{0}=(0,0,0)$, the Jacobian matrix is defined as

$$
A=\left(\begin{array}{ccc}
a & b & 0 \\
-b & a & d \cos z \\
e \cos x & 0 & c
\end{array}\right)_{(0,0,0)}=\left(\begin{array}{ccc}
a & b & 0 \\
-b & a & d \\
e & 0 & c
\end{array}\right) .
$$

The characteristic polynomial of the Jacobian matrix of system (3) at $E_{0}$ has the form

$$
\begin{aligned}
p(\lambda)= & \lambda^{3}-(2 a+c) \lambda^{2}+\left(a^{2}+b^{2}+2 a c\right) \lambda \\
& -\left(a^{2}+b^{2}\right) c-b d e .
\end{aligned}
$$




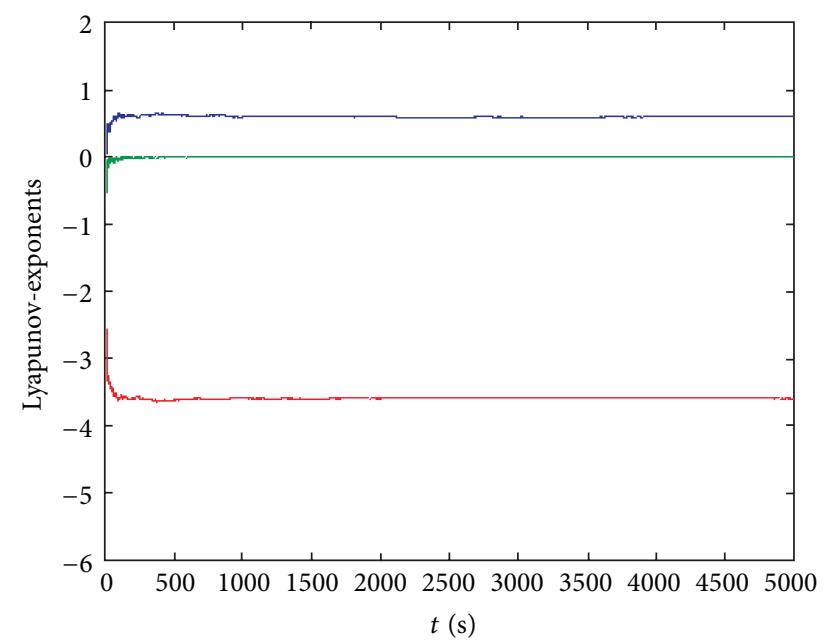

(a)

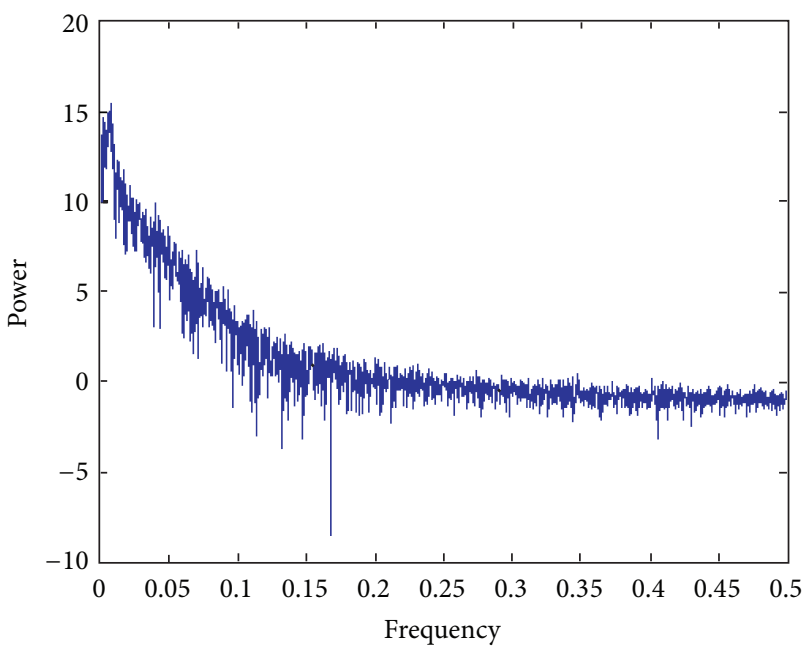

(c)

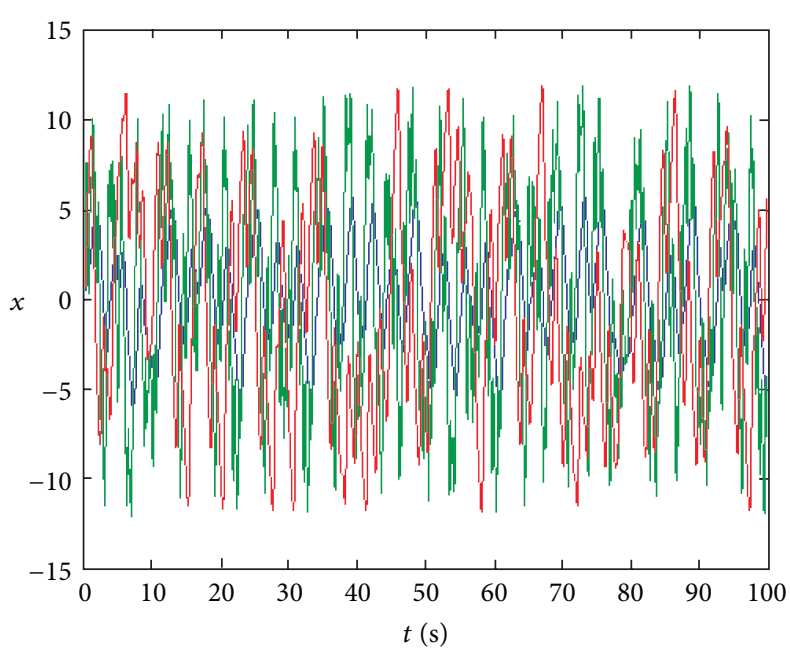

(b)

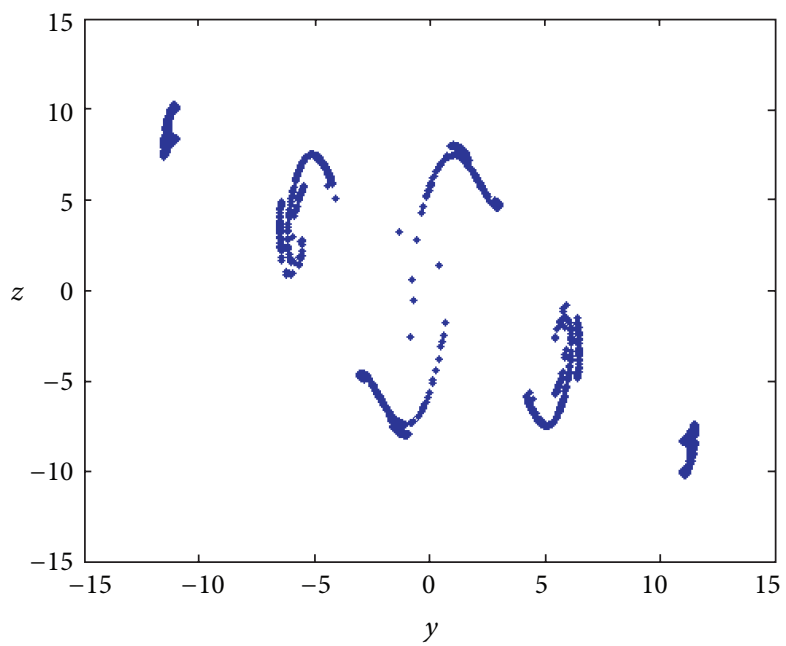

(d)

Figure 2: (a) Time history, (b) frequency spectrum, (c) Poincaré mapin $y-z\{x=0\}$ plane, and (d) Lyapunov-exponent spectrum of chaotic system (3).

From Lemma 1 the equilibrium $E_{0}=(0,0,0)$ is locally asymptotically stable if the coefficients of the characteristic polynomial satisfy

$$
\begin{gathered}
-\left(a^{2}+b^{2}\right) c-b d e>0,\left(a^{2}+b^{2}+2 a c\right)>0,-(2 a+c)>0, \\
-(2 a+c)\left(a^{2}+b^{2}+2 a c\right)>\left(-\left(a^{2}+b^{2}\right) c-b d e\right) .
\end{gathered}
$$

The last inequality in (9) can be written as $d>\left(2 a^{3}+2 a b^{2}+\right.$ $\left.4 a^{2} c+2 a c^{2}\right) / b e$. So if $(a, b, c, d, e) \in w_{1}$ then the equilibrium $E_{0}$ is locally asymptotically stable and if $(a, b, c, d, e) \in w_{2}$ then the equilibrium $E_{0}$ is unstable. This proves items 1 and 2 of the proposition. Item 3 of the proposition will be proved in the next section (see Theorem 3) with the calculation of the first Lyapunov coefficient at the equilibrium $E_{0}$ for parameters in $w_{3}$. In fact, when $(a, b, c, d, e) \in w_{3}$ the equilibrium $E_{0}$ is a Hopf point; that is, the characteristic polynomial of the Jacobian matrix of system (3) at $E_{0}$ has eigenvalues of the form

$$
\lambda_{1}=2 a+c, \quad \lambda_{2,3}= \pm i \omega_{0}= \pm i \sqrt{a^{2}+b^{2}+2 a c} .
$$

Define

$$
d_{0}=\frac{\left(2 a^{3}+2 a b^{2}+4 a^{2} c+2 a c^{2}\right)}{b e} .
$$

If $(a, b, c, d, e) \in w_{3}$ then the Jacobian matrix of (4) at $E_{0}$ has one negative real eigenvalue $\lambda_{1}$ and a pair of purely imaginary eigenvalues $\lambda_{2,3}$. From the center manifold theorem, at a Hopf point a two-dimensional center manifold is well-defined; it is invariant under the flow generated by (3) and can be continued with arbitrary high class of differentiability to nearby parameter values. This center manifold is attracting since $\lambda_{0}$. So it is enough to study the stability of $E_{0}$ for the flow restricted to the family of parameter-dependent continuations of the center manifold. 


\section{Hopf Bifurcation Analysis in System (4)}

This section is a review of the projection method described in $[20,21]$ for the calculation of the first Lyapunov coefficients associated with Hopf bifurcation, denoted by $l_{1}$.

Consider the differential equation

$$
x^{\prime}=f(x, \zeta)
$$

where $x \in R^{3}, \zeta \in R^{5}$ are, respectively, vectors representing phase variables and control parameters. Assume that $f$ is of class $C^{\infty}$ in $R^{3} \times R^{5}$. Suppose that (12) has an equilibrium point $x=x_{0}$ at $\zeta=\zeta_{0}$ and, denoting the variable $x-x_{0}$ also by $x$, write

$$
F(x)=f\left(x, \zeta_{0}\right)
$$

as

$$
F(x)=A x+\frac{1}{2} B(x, x)+\frac{1}{6} C(x, x, x)+O\left(\|x\|^{4}\right),
$$

where $A=f_{x}\left(0, \zeta_{0}\right)$, and for $i=1,2,3$,

$$
\begin{aligned}
B_{i}(x, y) & =\left.\sum_{j, k=1}^{3} \frac{\partial^{2} F_{i}(\xi)}{\partial \xi_{i} \partial \xi_{k}}\right|_{\xi=0} x_{i} y_{k}, \\
C_{i}(x, y, z) & =\left.\sum_{j, k, l=1}^{3} \frac{\partial^{3} F_{i}(\xi)}{\partial \xi_{i} \partial \xi_{k} \partial \xi_{l}}\right|_{\xi=0} x_{i} y_{k} z_{l} .
\end{aligned}
$$

Suppose that $\left(x_{0}, \zeta_{0}\right)$ is an equilibrium point of (12) where the Jacobian matrix $A$ has a pair of purely imaginary eigenvalues $\lambda_{2,3}= \pm i \omega_{0},\left(\omega_{0}>0\right)$, and admits no other eigenvalue with zero real part. Let $T^{c}$ be the generalized eigenspace of $A$ corresponding to $\lambda_{2,3}$. By this it is meant the largest subspace invariant by $A$ on the largest subspace invariant the eigenvalues are $\lambda_{2,3}$.

Let $p, q \in C^{3}$ be vectors such that

$$
A q=i \omega_{0} q, \quad A^{T} p=-i \omega_{0} p, \quad\langle p, q\rangle=\sum_{i=1}^{3} \bar{p}_{i} q_{i}=1,
$$

where $A^{T}$ is the transpose of the matrix $A$ and $\bar{q}$ is the conjugate complex of the matrix $q$. Any vector $y \in T^{c}$ can be represented as $y=\omega q+\overline{\omega q}$, where $\omega=\langle p, y\rangle \in C$ and $\bar{\omega}$ is the conjugate complex of the matrix $\omega$. The two-dimensional center manifold associated with the eigenvalues $\lambda_{2,3}= \pm i \omega_{0}$ can be parameterized by the variables $\omega$ and $\bar{\omega}$ by means of an immersion of the form $x=H(\omega, \bar{\omega})$, where $H: C^{2} \rightarrow R^{3}$ has a Taylor expansion of the form

$$
H(\omega, \bar{\omega})=\omega q+\overline{\omega q}+\sum_{2 \leq j+k \leq 7} \frac{1}{j ! k !} h_{j k} \omega^{j} \bar{\omega}^{k}+O\left(|\omega|^{8}\right),
$$

with $h_{j k} \in C^{3}$, and $h_{j k}=\bar{h}_{k j}$. Substituting this expression into (12) we obtain the following differential equation:

$$
H_{\omega} \omega^{\prime}+H_{\bar{\omega}} \bar{\omega}^{\prime}=F(H(\omega, \bar{\omega}))
$$

where $F$ is given by (13). The complex vectors $h_{j k}$ are obtained solving the system of linear equations defined by the coefficients of (18), taking into account the coefficients of $F$, so that system (18), on the chart $\omega$ for a central manifold, writes as follows:

$$
\begin{gathered}
\omega^{\prime}=i \omega_{0} \omega+\frac{1}{2} G_{21} \omega|\omega|^{2}+\frac{1}{12} G_{32} \omega|\omega|^{4} \\
+\frac{1}{144} G_{43} \omega|\omega|^{6}+O\left(|\omega|^{8}\right)
\end{gathered}
$$

with $G_{j k} \in C$.

The first Lyapunov coefficient $l_{1}$ is defined by

$$
l_{1}=\frac{1}{2} \operatorname{Re} G_{21},
$$

where $G_{21}=\left\langle p, H_{21}\right\rangle, H_{21}=C(q, q, \bar{q})+B\left(\bar{q}, h_{20}\right)+2 B\left(q, h_{11}\right)$, $h_{20}=\left(2 i \omega_{0} I_{3}-A\right)^{-1} B(q, q), h_{11}=-A^{-1} B(q, \bar{q})$, and $I_{3}$ is the unit $3 \times 3$ matrix.

A Hopf point $\left(x_{0}, \zeta_{0}\right)$ of system (12) is an equilibrium point where the Jacobian matrix $A$ has a pair of purely imaginary eigenvalues $\lambda_{2,3}= \pm i \omega_{0},\left(\omega_{0}>0\right)$ and the other eigenvalue $\lambda_{1} \neq 0$. From the center manifold theorem, at a Hopf point a two-dimensional center manifold is welldefined; it is invariant under the flow generated by (12) and can be continued with arbitrary high class of differentiability to nearby parameter values.

A Hopf point is called transversal if the parameter dependent complex eigenvalues cross the imaginary axis with nonzero derivative. In a neighborhood of a transversal Hopf point with $l_{1} \neq 0$ the dynamic behavior of the system (12), reduced to the family of parameter-dependent continuations of the center manifold, is orbitally topologically equivalent to the following complex normal form $\omega^{\prime}=(\eta+i \omega) \omega+l_{1} \omega|\omega|^{2}$, where $\omega \in C$. $\eta, \omega$, and $l_{1}$ are real functions having derivatives of arbitrary higher order, which are continuations of $0, \omega_{0}$, and the first Lyapunov coefficient at the Hopf point. When $l_{1}<0\left(l_{1}>0\right)$ one family of stable (unstable) periodic orbits can be found on this family of manifolds, shrinking to an equilibrium point at the Hopf point.

Next we study the stability of $E_{0}$ for parameters in $H$. We have the following theorem.

Theorem 3. Consider the system (3); the first Lyapunov coefficient at $E_{0}$ for parameter values in $w_{3}$ is given by

$$
l_{1}\left(a, b, c, d_{0}, e\right)=\frac{e a \omega^{2}\left(k+e^{2}\right)-a^{2}\left(k-e^{2}\right)}{2 e^{3}\left(2 a^{2}+b^{2}+2 a c\right)},
$$

where

$$
k=a^{2}+b^{2}+c^{2}+2 a c, \quad \omega=\sqrt{a^{2}+b^{2}+2 a c} .
$$

As $b>e>0$ then $l_{1}\left(a, b, c, d_{0}, e\right)<0$ and system (3) has a transversal Hopf point at $E_{0}$ for $d=d_{0}$ and $b>e>0$ (see Figure 4). More precisely, Hopf point at $E_{0}$ is stable and for each $d>d_{0}$, but close to $d_{0}$, there exists a stable periodic orbit near the asymptotically stable equilibrium point $E_{0}$. 


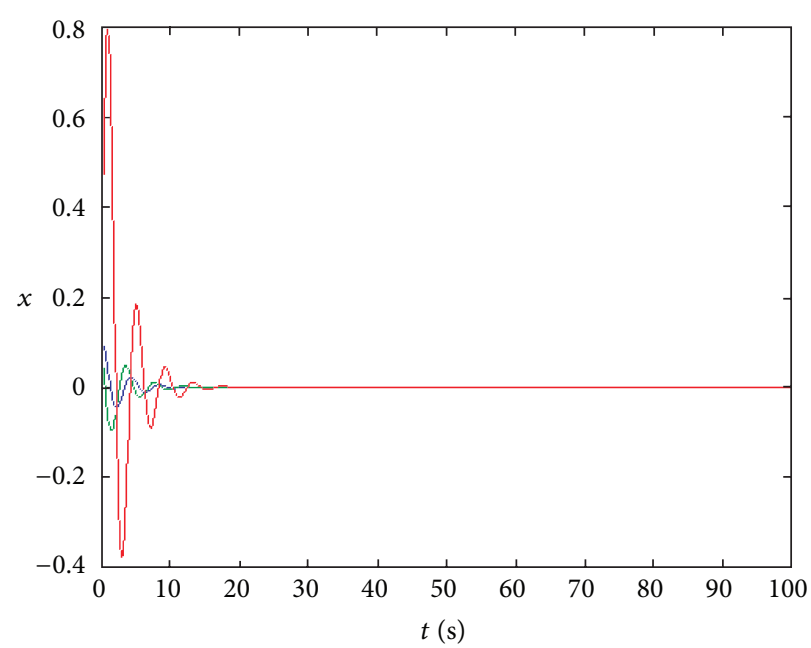

(a)

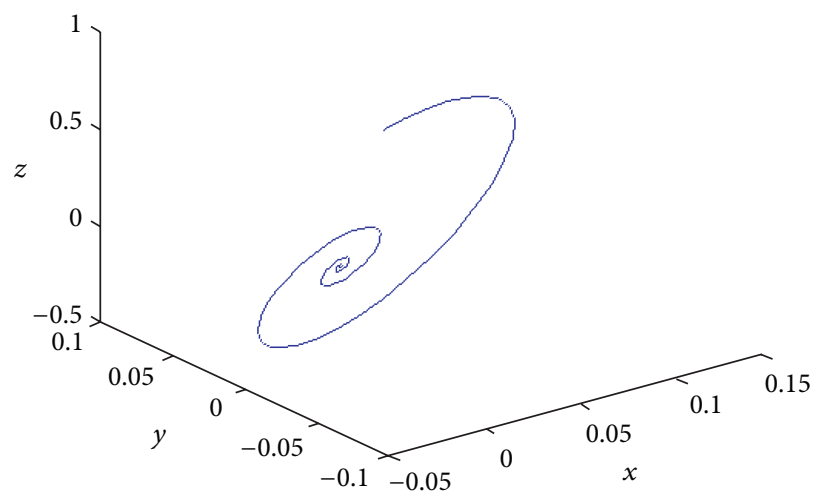

(b)

Figure 3: Time history and phase diagram of system (3) with $a=-1, b=1, c=-1, e=18$, and $d=-0.2$.

Proof. For parameters on the Hopf surface $w_{3}$ one has

$$
\begin{gathered}
\lambda_{1}=2 a+c, \quad \lambda_{2,3}= \pm i \omega_{0}= \pm i \sqrt{a^{2}+b^{2}+2 a c}, \\
q=\left(\frac{i \sqrt{a^{2}+b^{2}+2 a c}-c}{e},\right. \\
\left.\frac{\left(i \sqrt{a^{2}+b^{2}+2 a c}-a\right)\left(i \sqrt{a^{2}+b^{2}+2 a c}-c\right)}{b e}, 1\right), \\
p=\left(p_{1}, p_{2}, p_{3}\right),
\end{gathered}
$$

$C(q, q, \bar{q})$

$$
=\left(0,-d, \frac{\left(i \sqrt{a^{2}+b^{2}+2 a c}-c\right)^{2}\left(i \sqrt{a^{2}+b^{2}+2 a c}+c\right)}{e^{2}}\right),
$$

where

$$
\begin{aligned}
& p_{1}=\frac{e\left(\left(i \sqrt{a^{2}+b^{2}+2 a c}+a\right)\left(i \sqrt{a^{2}+b^{2}+2 a c}+c\right)\right)}{-2\left(a^{2}+b^{2}+c^{2}+2 a c\right) \sqrt{a^{2}+b^{2}+2 a c} i-b d e} \\
& p_{2}=\frac{b e\left(\left(i \sqrt{a^{2}+b^{2}+2 a c}+c\right)\right)}{-2\left(a^{2}+b^{2}+c^{2}+2 a c\right) \sqrt{a^{2}+b^{2}+2 a c} i-b d e} \\
& p_{3}=\frac{b d e}{-2\left(a^{2}+b^{2}+c^{2}+2 a c\right) \sqrt{a^{2}+b^{2}+2 a c} i-b d e}
\end{aligned}
$$

We can get

$$
\begin{aligned}
H_{21}= & (0,-d, \\
& \left.\frac{\left(i \sqrt{a^{2}+b^{2}+2 a c}-c\right)^{2}\left(i \sqrt{a^{2}+b^{2}+2 a c}+c\right)}{e^{2}}\right),
\end{aligned}
$$

$$
\begin{aligned}
G_{21}= & \left\langle p, H_{21}\right\rangle \\
= & \left(-2 b d e \omega^{2} k\left(k+e^{2}\right)+b^{2} c d^{2} e\left(k-e^{2}\right)\right. \\
& \left.-\left[2 b c d e k\left(k-e^{2}\right)+b d e^{2} b d\left(k+e^{2}\right)\right] \omega i\right) \\
& \times\left(-(2 e k \omega)^{2}-\left(b d e^{2}\right)^{2}\right)^{-1}
\end{aligned}
$$

where

$$
k=a^{2}+b^{2}+c^{2}+2 a c, \quad \omega=\sqrt{a^{2}+b^{2}+2 a c} .
$$

Now it remains only to verify the transversality condition of the Hopf bifurcation, so consider the family of differential equation (3) regarded as dependent on the parameter $d$. The real part $\xi$ of the pair of complex eigenvalues at the critical parameter $d=d_{0}$ verifies

$$
\xi^{\prime}\left(d_{0}\right)=\operatorname{Re}\left\langle p,\left.\frac{d A}{d d}\right|_{d=d_{0}} q\right\rangle=-\frac{b e \omega^{2}+a b c e}{2 k \omega+2 a^{2} k}<0 .
$$

Since $\xi^{\prime}\left(d_{0}\right) \neq 0$, the transversality condition at the Hopf point holds.

\section{Numerical Example}

Next, we will give a numerical example of system (3). Let $a=-1, b=1, c=-1$, and $e=18$; we can compute the Hopf bifurcation value $d_{0}=-0.555556$. The equilibrium is stable when $d=-0.2>d_{0}$ and unstable when $d=-0.8<d_{0}$, as shown in Figure 3 and Figure 5, respectively. Thus, the periodic solutions bifurcating from the equilibrium point $E_{0}$ are supercritical and stable.

The characteristic polynomial of the Jacobian matrix of system (3) at $E_{0}$ has the form

$$
p(\lambda)=\lambda^{3}+A \lambda^{2}+B \lambda+C,
$$




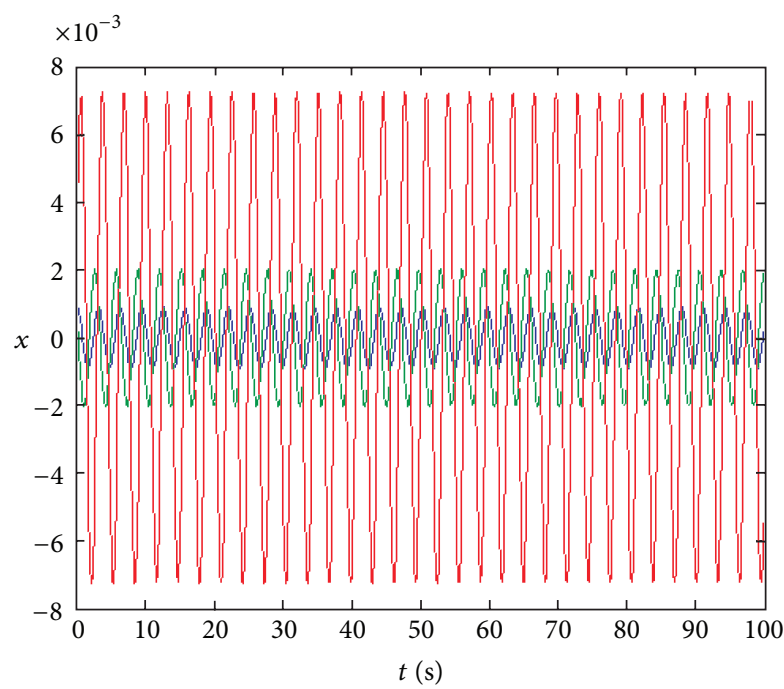

(a)

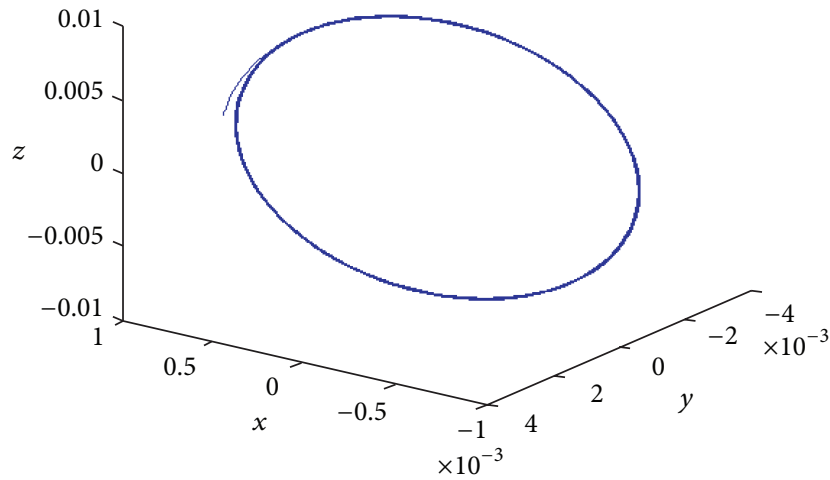

(b)

FIgURE 4: Time history and phase diagram of system (3) with $a=-1, b=1, c=-1, e=18$, and $d=-0.555556$.

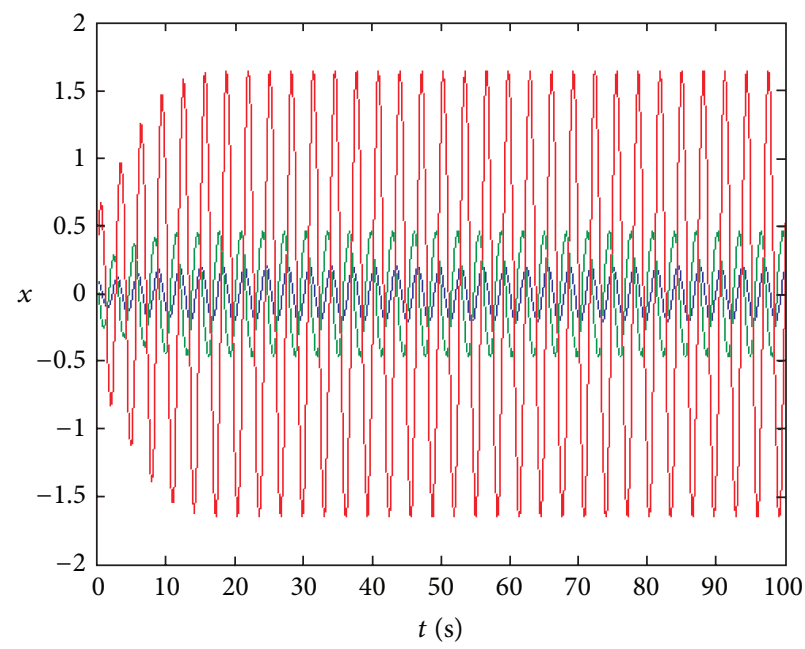

(a)

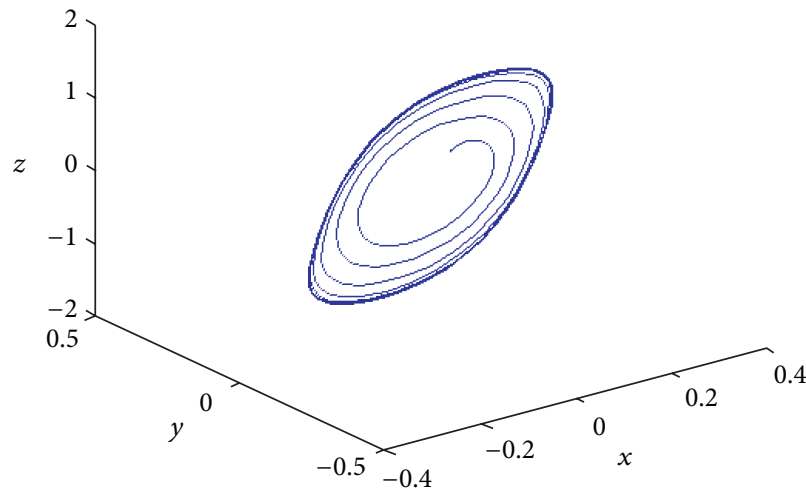

(b)

FIGURE 5: Time history and phase diagram of system (3) with $a=-1, b=1, c=-1, e=18$, and $d=-0.8$.

where

$$
\begin{aligned}
& A=-2 a-c, \quad B=a^{2}+b^{2}+2 a c, \\
& C=-c\left(a^{2}+b^{2}\right)-b d e .
\end{aligned}
$$

If $A>0, B>0, C>0$, and $A B-C>0$, then the equilibrium $E_{0}$ is asymptotically stable. If $A>0, B>0, C>0$, and $A B-C=0$, then the characteristic polynomial of the Jacobian matrix of system(1) at $E_{0}$ with one pair of conjugate nonzero purely imaginary eigenvalues and the real part of other characteristic root are less than zero.

When the parameters $c=-1, d=36$, and $e=18$ are fixed while parameters $a, b$ are varied, some different dynamical behaviors of system ( 3 ) are obtained. Let $A=0, B=0, C=0$, and $A B-C=0$, and use MATLAB to draw the stability region on the parameter plane $a-b$, as shown in Figure 6. In this figure, the symbol $L_{i}, i=1,2,3,4$, represents $A=0, B=0$, $C=0$, and $A B-C=0$, respectively. And in region (I), $A>0$, $B<0, C<0, A B-C>0$, in region (II), $A>0, B<0$, $C>0, A B-C<0$, in region (III), $A<0, B<0, C<0$, $A B-C>0$, in region (IV): $A<0, B<0, C>0, A B-C<0$, in the region (V): $A<0, B>0, C>0, A B-C<0$, in the region (VI), $A<0, B>0, C<0, A B-C>0$, in region (VII), $A<0, B>0, C<0, A B-C<0$, in region (VIII), $A>0$, $B>0, C<0, A B-C>0$, in region (IX), $A>0, B>0$, $C>0, A B-C<0$, and in region (X), $A>0, B>0, C>0$, $A B-C>0$. All of the points are stable in region $(\mathrm{X})$ and in the other regions are unstable.

According to the above analysis, we know the $L_{4}$ is the Hopf bifurcation boundaries of the system. And the system 


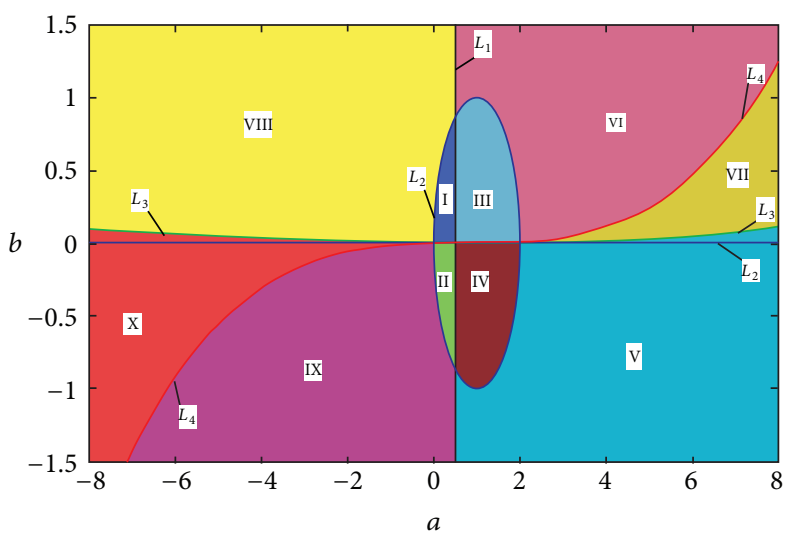

FIgURE 6: The stable region on the parameter plane $(a, b)$.

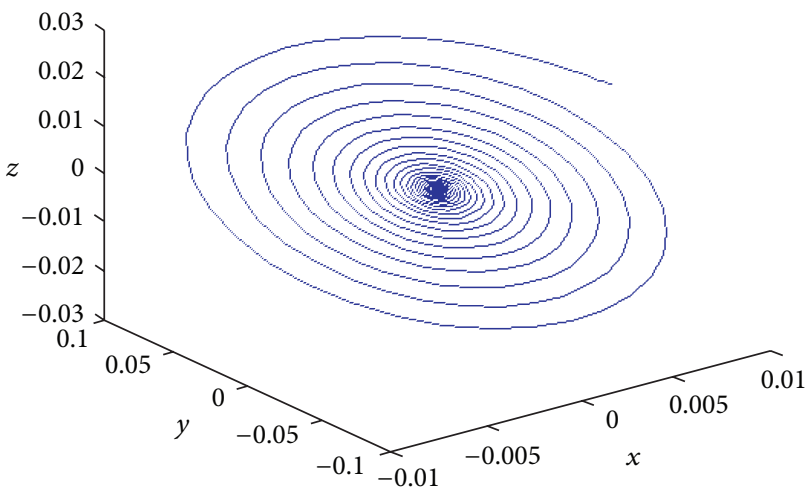

(a) $a=-6, b=-0.8$

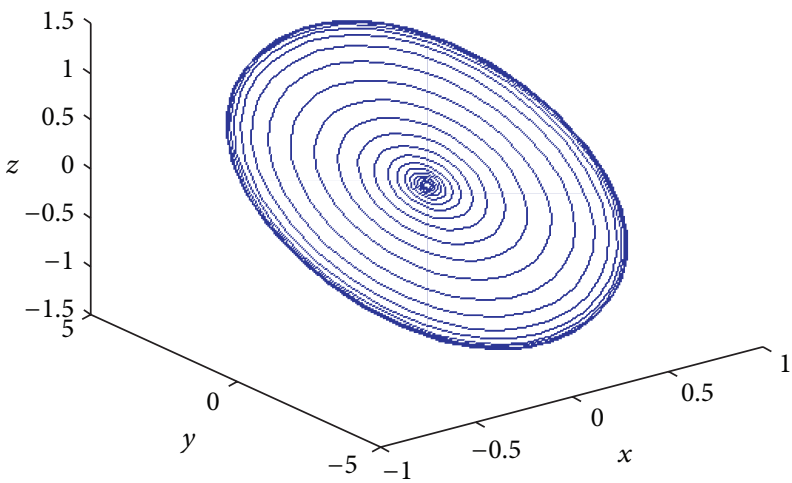

(c) $a=-6, b=-1.2$

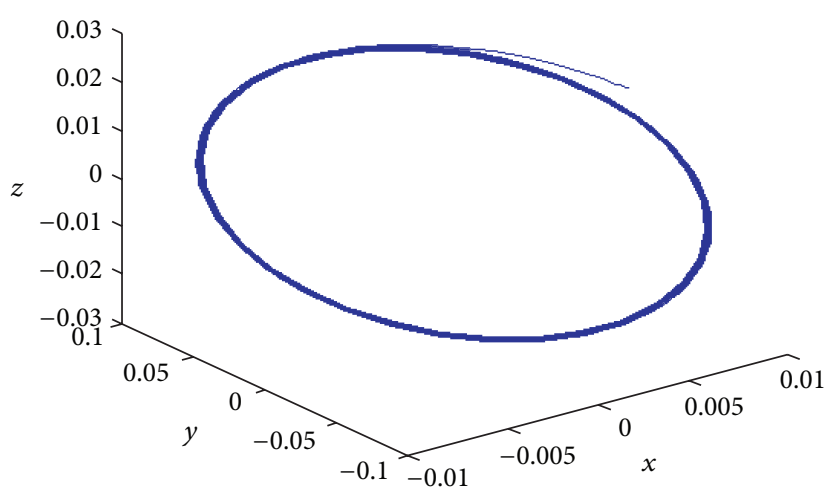

(b) $a=-6, b=-0.9232$

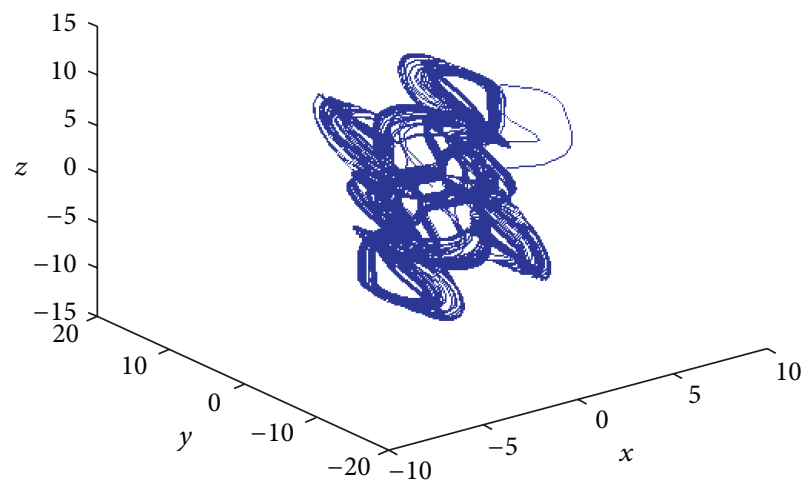

(d) $a=-1, b=1$

FIGURE 7: Phase diagram of system (3) with different parameters.

(3) will generate the Hopf bifurcation when the bifurcation parameter passes through $L_{4}$ from region (X) to region (IX). Next, we combined with Figure 6 and fixed $a=-6$; then we can compute the Hopf bifurcation value $b_{0}=-0.9232$. The system (3) has a transversal Hopf point at $E_{0}$ when the bifurcation parameter $b$ passes through $b_{0}$, as shown in Figure $7(\mathrm{~b})$. In addition, we choose $(a, b)=(-6,-0.8)$, $(a, b)=(-6,-1.2)$, and $(a, b)=(-1,1)$; the points are located in region (X), region (IX), and region (VIII), respectively, as shown in Figures 7(a) and 7(c) and Figure 7(d).
When the parameters $a=-1, d=36$, and $e=18$ are fixed while parameters $b, c$ are varied, some different dynamical behaviors of system (3) are obtained. Let $A=0, B=0$, and $C=0, A B-C=0$, and use MATLAB to draw the stability region on the parameter plane $b-c$, as shown in Figure 8. In this figure, the symbol $L_{i}, i=1,2,3,4$, represents $A=0$, $B=0, C=0$, and $A B-C=0$, respectively. And in region (I), $A>0, B<0, C>0, A B-C<0$, in region (II), $A>0$, $B<0, C<0, A B-C>0$, in region (III), $A<0, B<0$, $C<0, A B-C>0$, in region (IV), $A<0, B<0, C>0$, 


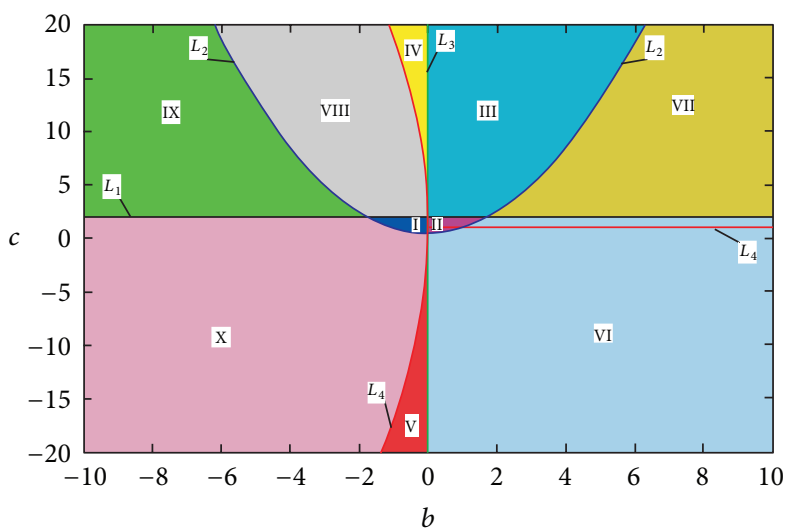

FIGURE 8: The stable region on the parameter plane $(b, c)$.

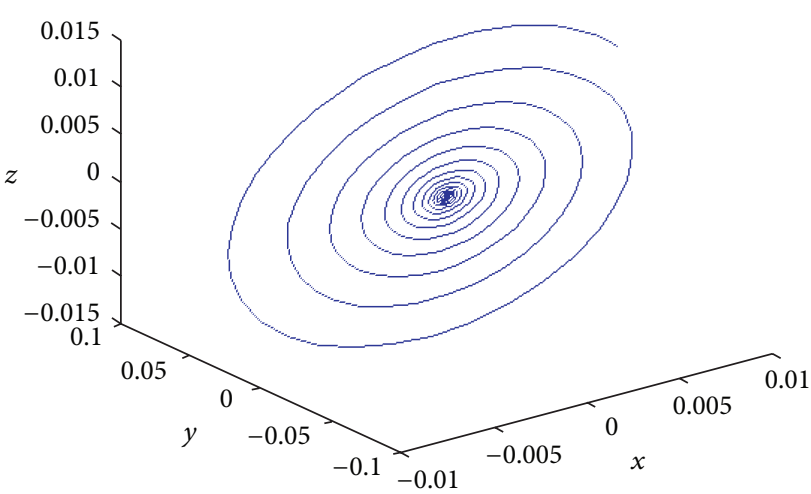

(a) $b=-0.5, c=-14$

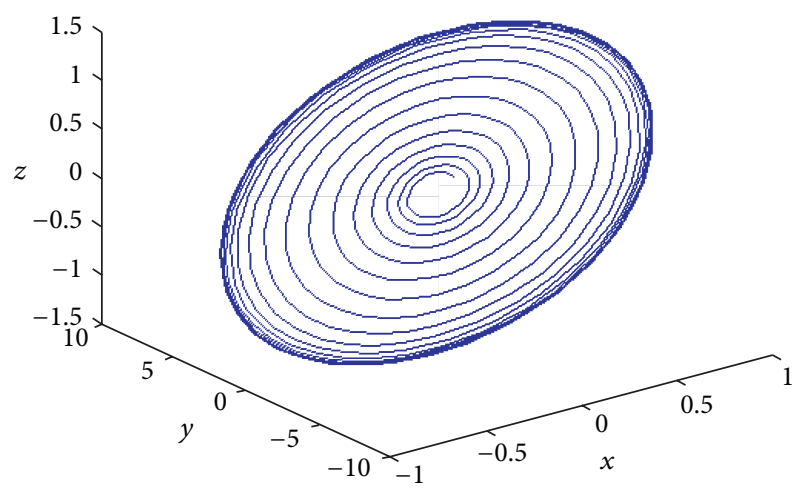

(c) $b=-0.5, c=-10$

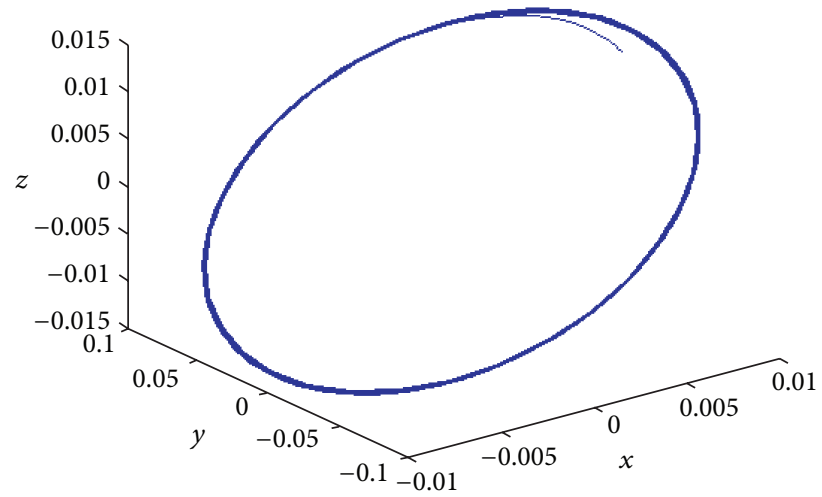

(b) $b=-0.5, c=-11.7181$

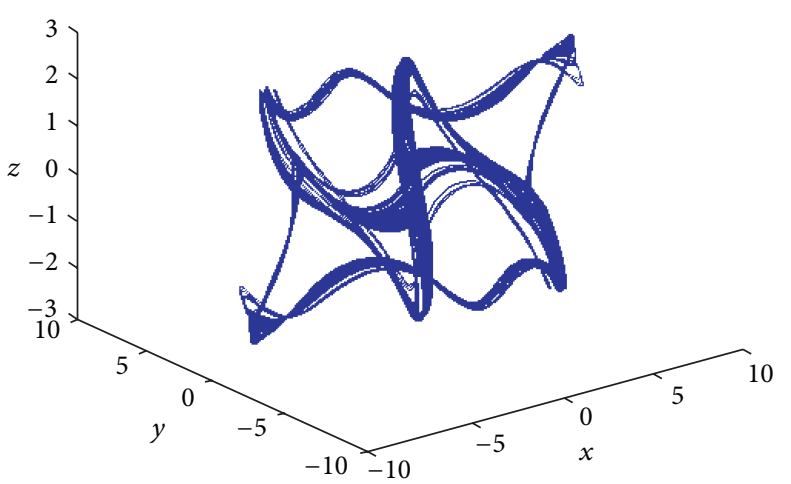

(d) $b=6, c=-5$

FiguRE 9: Phase diagram of system (3) with different parameters.

$A B-C>0$, in region (V), $A>0, B>0, C>0, A B-C>0$, in region (VI), $A>0, B>0, C<0, A B-C>0$, in region (VII), $A<0, B>0, C<0, A B-C>0$, in region (VIII), $A<0, B<0, C>0, A B-C>0$, in region (IX), $A<0, B>0$, $C>0, A B-C<0$, and in region (X), $A>0, B>0, C>0$, $A B-C<0$. All of the points are stable in region $(\mathrm{V})$ and in the other regions are unstable.

According to the above analysis, we know the $L_{4}$ is the Hopf bifurcation boundaries of the system. And the system (3) will generate the Hopf bifurcation when the bifurcation parameter passes through $L_{4}$ from region (V) to region (X). Next, we combined with Figure 8 and fixed $b=-0.5$; then we can compute the Hopf bifurcation value $c_{0}=-11.7181$. The system (3) has a transversal Hopf point at $E_{0}$ when the bifurcation parameter $c$ passes through $c_{0}$, as shown in Figure $9(\mathrm{~b})$. In addition, we choose $(b, c)=(-0.5,-10)$, $(b, c)=(-0.5,-14)$, and $(b, c)=(6,-5)$; the points are located in region $(\mathrm{V})$, region $(\mathrm{X})$, and region $(\mathrm{VI})$, respectively, as shown in Figures 9(a), 9(c), and 9(d).

\section{Sliding Mode Control of Chaotic Vibrations}

6.1. The Design of the Controller. We designed a sliding surface with good nature and made the system possess 


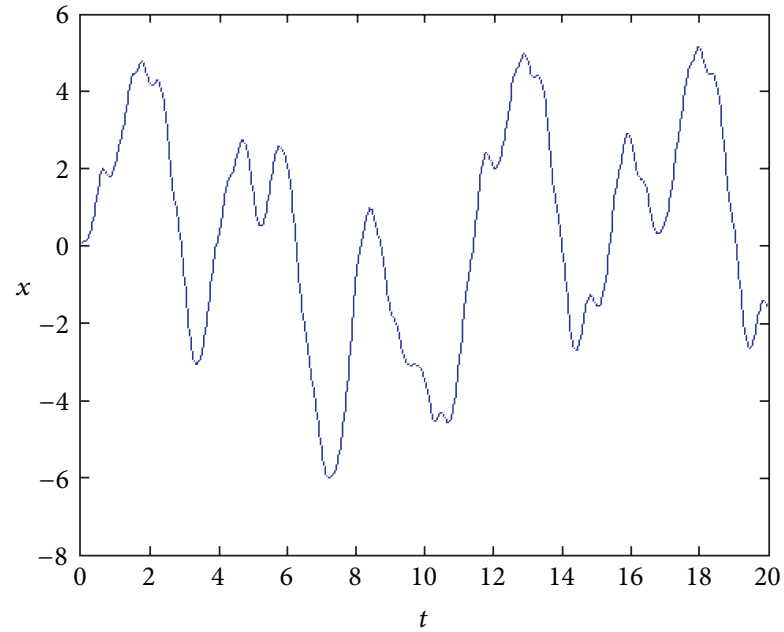

(a) Time domain chart of $x$ before control

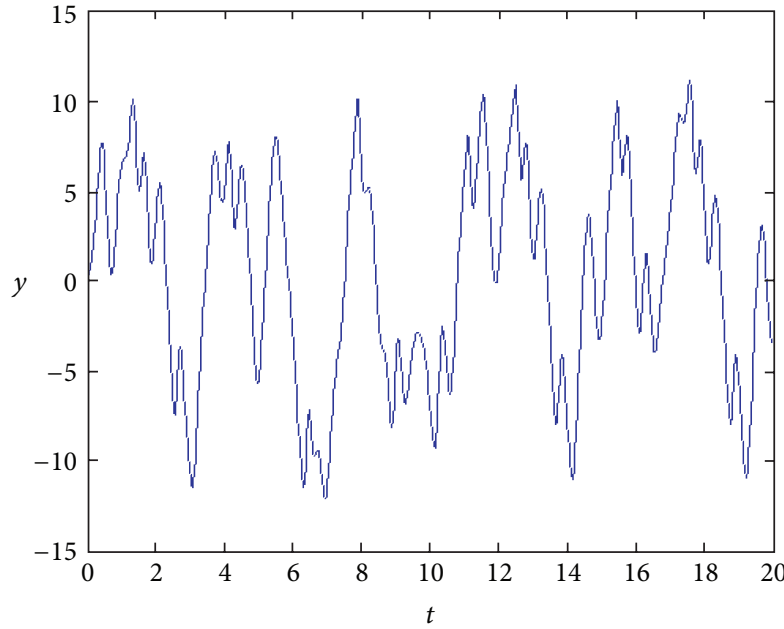

(b) Time domain chart of $y$ before control

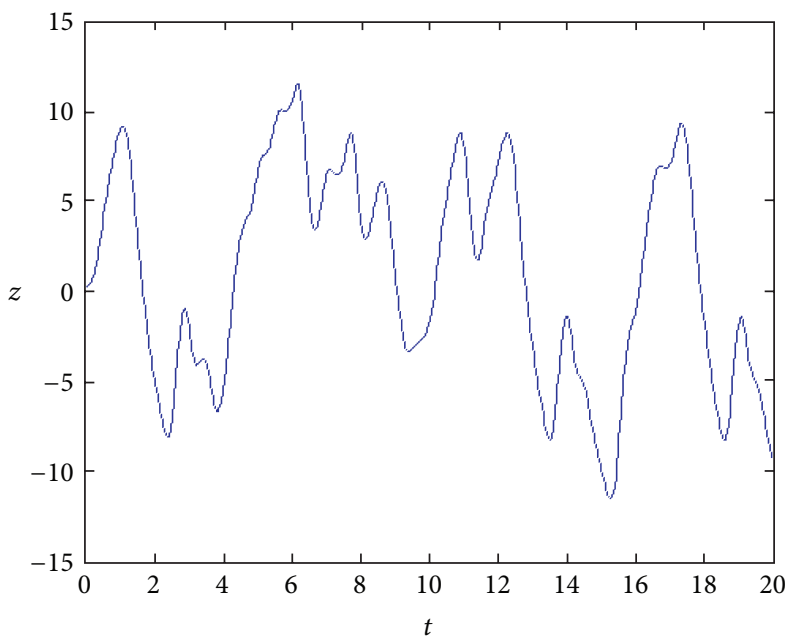

(c) Time domain chart of $z$ before control

FIGURE 10: Time domain charts of state variables before control.

the desired properties when it has limitations on the sliding surface. Then to facilitate control, make the system reach the sliding surface and keep sliding. After joining the controller, the system (3) has the following form:

$$
\begin{gathered}
\dot{x}=a x+b y+d_{1}+u_{1}, \\
\dot{y}=-b x+a y+d \sin z+d_{2}+u_{2}, \\
\dot{z}=c z+e \sin x+d_{3}+u_{3},
\end{gathered}
$$

where $u_{1}, u_{2}$, and $u_{3}$ are control inputs. If we join the reasonable controller, we can control the chaos system to the required range or the fixed point.
Define the following matrix:

$$
\begin{aligned}
& \mathbf{A}=\left(\begin{array}{ccc}
-1 & 1 & 0 \\
-1 & -1 & 0 \\
0 & 0 & 4
\end{array}\right), \quad \mathbf{B}=\left(\begin{array}{lll}
1 & 0 & 0 \\
0 & 1 & 0 \\
0 & 0 & 1
\end{array}\right), \\
& \mathbf{d}=\left(\begin{array}{l}
d_{1} \\
d_{2} \\
d_{3}
\end{array}\right), \quad \mathbf{g}=\left(\begin{array}{c}
0 \\
36 \sin z \\
18 \sin x
\end{array}\right),
\end{aligned}
$$

where $\mathbf{A}$ is the linear matrix of the system, $\mathbf{B}$ is the bounded perturbation matrix, $\mathbf{d}$ is the control matrix, and $\mathbf{g}$ is the nonlinear matrix of the system. The purpose of control is to let the system state $\mathbf{x}=\left[x_{1}, x_{2}, x_{3}\right]^{T}$ to track a time-varying state $\mathbf{x}_{d}=\left[x_{d 1}, x_{d 2}, x_{d 3}\right]^{T}$. So, we can define the tracking error

$$
\mathbf{e}=\mathbf{x}-\mathbf{x}_{d}
$$




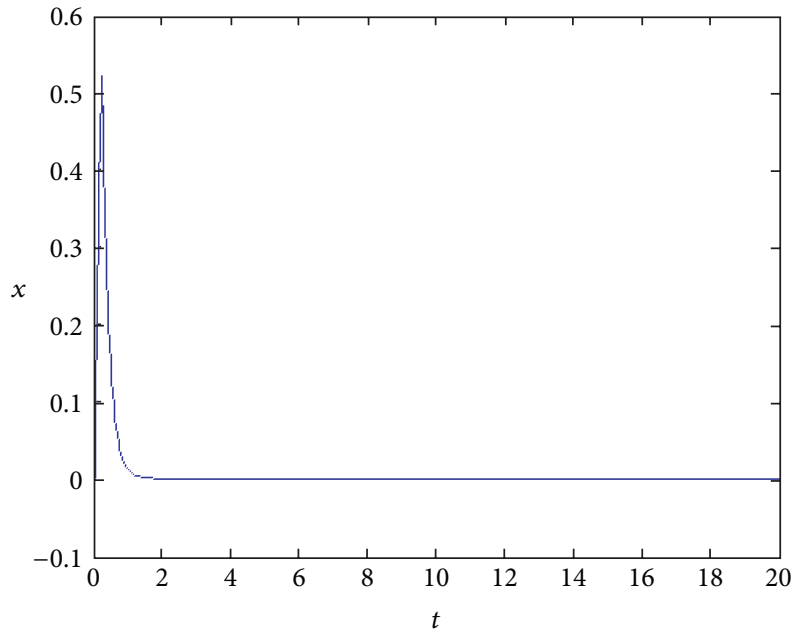

(a) Time domain chart of $x$ after control

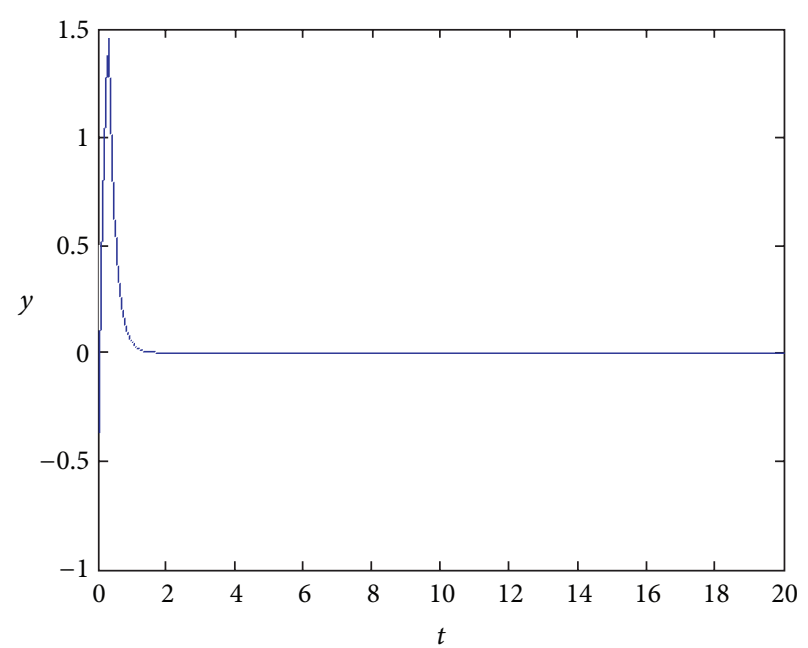

(b) Time domain chart of $y$ after control

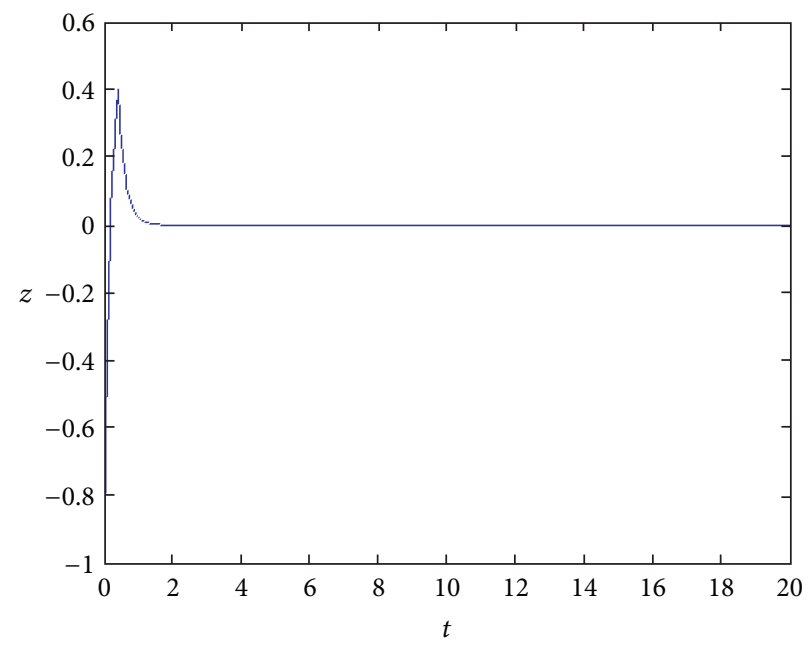

(c) Time domain chart of $z$ after control

FIgURE 11: Time domain charts of state variables after control.

The error dynamic system can be written as

$$
\dot{\mathbf{e}}=\dot{\mathbf{x}}-\dot{\mathbf{x}}_{d}=\mathbf{A x}+\mathbf{B g}+\mathbf{B u}+\mathbf{d}-\dot{\mathbf{x}}_{d}
$$

Define the time-varying proportional integral sliding mode surface

$$
\mathbf{S}=\mathbf{K e}-\int_{0}^{t} \mathbf{K}(\mathbf{A}-\mathbf{B} \mathbf{L}) \mathbf{e}(\tau) d \tau
$$

where $\mathbf{K} \in \mathbf{R}^{3 \times 3}, \operatorname{det}(\mathbf{K B}) \neq 0$. For the convenience of calculation, we get $\mathbf{K}=\operatorname{diag}(1,1,1)$. The additional matrix $\mathbf{L} \in \mathbf{R}^{3 \times 3}$ and $\mathbf{A}-\mathbf{B L}$ is negative definite matrix. The equation $\mathbf{S}=\dot{\mathbf{S}}=0$ must be satisfied under the sliding mode, where

$$
\dot{\mathbf{S}}=\mathbf{K B g}+\mathbf{K B L e}+\mathbf{K B u}+\mathbf{K d}+\mathbf{K A x} \mathbf{x}_{d}-\mathbf{K}_{d} .
$$

In order to satisfy the sliding conditions, the following controller is designed:

$$
\begin{aligned}
& \mathbf{u}=- {[\mathbf{g}+\mathbf{L e}]-(\mathbf{K B})^{-1}\left[\mathbf{K A x} \mathbf{x}_{d}-\mathbf{K} \dot{\mathbf{x}}_{d}\right] } \\
&-(\mathbf{K B})^{-1}[\varepsilon+\|\mathbf{K B g}\|] \operatorname{sign}(\mathbf{S}),
\end{aligned}
$$

where $\operatorname{sign}(\mathbf{S})$ is symbolic function.

Proposition 4. The controller (36) can make the system (28) reach the sliding mode $\mathbf{S}=0$ in a limited time if the constant $\varepsilon$ satisfied the inequality $\varepsilon>\delta_{1}+\delta_{2}+1$, where $\delta_{1}, \delta_{2}$ are an arbitrary small positive numbers. The state variables and the selected reference state $\mathbf{x}_{d}$ are identical.

Proof. Construct the Lyapunov function $V=\mathbf{S}^{T} \mathbf{S}=\sum_{i=1}^{3} \mathbf{S}_{i}^{2}$; according to (34), (35), and (36) one has

$$
\begin{aligned}
\mathbf{S}^{T} \dot{\mathbf{S}} & =\mathbf{S}^{T}\left(\mathbf{K B g}+\mathbf{K B L e}+\mathbf{K B u}+\mathbf{K d}+\mathbf{K A} \mathbf{x}_{d}-\mathbf{K} \dot{\mathbf{x}}_{d}\right) \\
& =\mathbf{S}^{T}[\mathbf{K d}-(\varepsilon+\|\mathbf{K B g}\|) \operatorname{sign}(\mathbf{S})] \leq \mathbf{S}^{T}[\mathbf{d}-\varepsilon \operatorname{sign}(\mathbf{S})]
\end{aligned}
$$




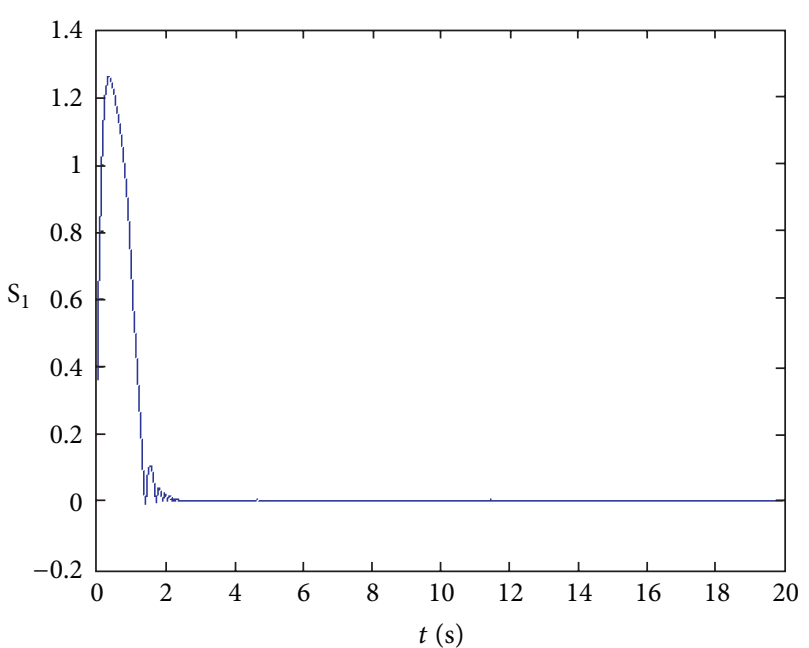

(a) Time domain chart of $S_{1}$ after control

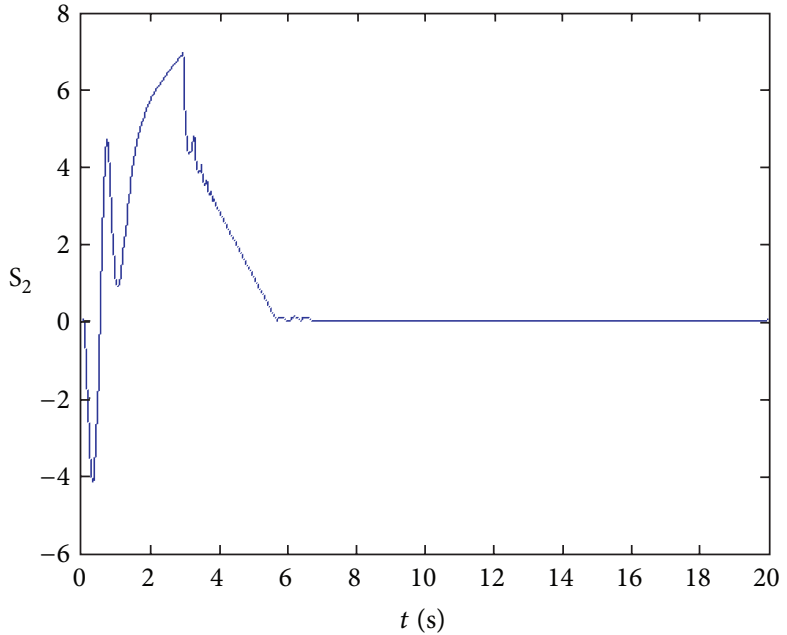

(b) Time domain chart of $S_{2}$ after control

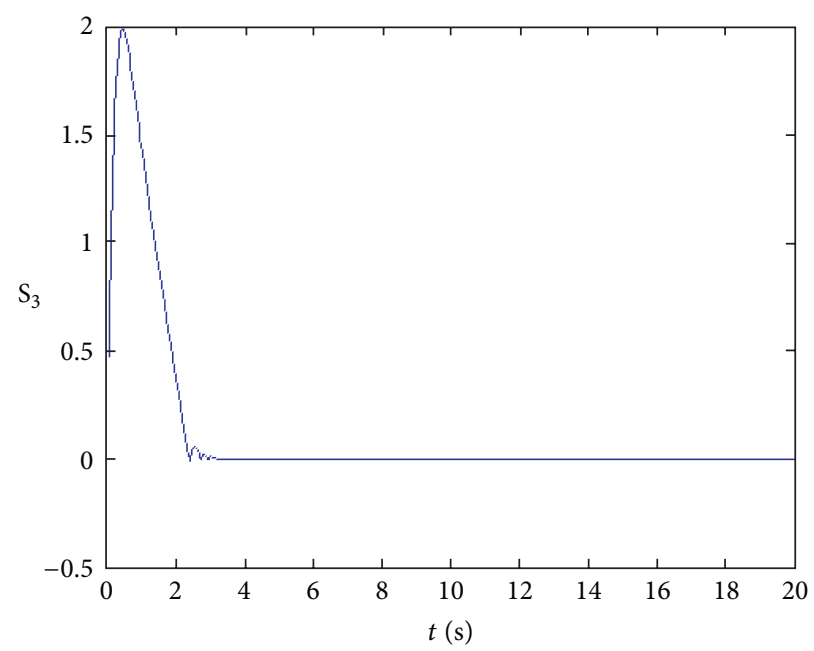

(c) Time domain chart of $S_{3}$ after control

FIGURE 12: Time domain charts of sliding surfaces after control.

$$
\begin{aligned}
& \leq \sum_{i=1}^{3}\left|\mathbf{S}_{i}\right| \delta_{1}+\sum_{i=1}^{3}\left|\mathbf{S}_{i}\right| \delta_{2}-\sum_{i=1}^{3}\left|\mathbf{S}_{i}\right| \varepsilon \\
& =\left|\delta_{1}+\delta_{2}-\varepsilon\right| \sum_{i=1}^{3}\left|\mathbf{S}_{i}\right|<-\sum_{i=1}^{3}\left|\mathbf{S}_{i}\right|
\end{aligned}
$$

By the same token, we get

$$
\dot{\mathbf{S}}^{T} \mathbf{S}<-\sum_{i=1}^{3}\left|\mathbf{S}_{i}\right|, \quad \dot{V}=\dot{\mathbf{S}}^{T} \mathbf{S}+\mathbf{S}^{T} \dot{\mathbf{S}}<-2 \sum_{i=1}^{3}\left|\mathbf{S}_{i}\right|
$$

So the proposition follows.

6.2. The Numerical Simulation. In the case of $u_{1}=u_{2}=u_{3}=$ 0 , the time-domain charts of the state variables of system (28) are shown in Figure 10. Figure 10 illustrates that the system (28) has a periodic motion state before control.
In order to control the system (28) to the target state, we select the eigenvalue of $\mathbf{A}-\mathbf{B L}$ to be $\mathbf{P}=[-5,-5,-5]$. The pole-placement method is adopted to get the following matrix:

$$
\mathbf{L}=\left(\begin{array}{ccc}
4 & 1 & 0 \\
-1 & 4 & 0 \\
0 & 0 & 4
\end{array}\right)
$$

Select proportional integral sliding mode surface as follows:

$$
\begin{aligned}
& S_{1}=e_{1}+\int_{0}^{t} 5 e_{1}(\tau) d \tau, \\
& S_{2}=e_{2}+\int_{0}^{t} 5 e_{2}(\tau) d \tau, \\
& S_{3}=e_{3}+\int_{0}^{t} 5 e_{3}(\tau) d \tau .
\end{aligned}
$$


Set the initial value $\left[x_{1}(0), x_{2}(0), x_{3}(0)\right]=[0.1,0.1,0.1]$, and the reference state $x_{d 1}=x_{d 2}=x_{d 3}=x_{d}$. The control signal is as follows:

$$
\begin{aligned}
u_{1}= & -4 e_{1}-e_{2}+\dot{x}_{d}-\varepsilon \operatorname{sign}\left(S_{1}\right), \\
u_{2}= & -36 \sin z+e_{1}-4 e_{2}+2 x_{d}+\dot{x}_{d} \\
& -(\varepsilon+|36 \sin z|) \operatorname{sign}\left(S_{2}\right), \\
u_{3}= & -18 \sin x-4 e_{3}+x_{d}+\dot{x}_{d}-(\varepsilon+|18 \sin x|) \operatorname{sign}\left(S_{3}\right) .
\end{aligned}
$$

6.3. Control to the Fixed Point. We can stabilize the system (28) to any point by this method. In this paper, we select the fixed point $[0,0,0]$, reference state $\mathbf{x}_{d}=0$, small parameter $\varepsilon=7$, and the initial value of the sliding mode surface $\left[S_{1}(0), S_{2}(0), S_{3}(0)\right]=[0.1,0.1,0.1]$. The controller $\mathbf{u}(t)$ is activated at $t=1 \mathrm{~s}$, the time domain charts of state variables, and sliding surfaces as shown in Figure 11 and Figure 12, respectively.

Figures 11 and 12 show that, after joining the controller, the system (28) tracks to reference state $[0,0,0]$ ultimately, and the sliding mode surface $\mathbf{S}$ becomes 0 . It is proven that the system (28) reached the sliding mode.

\section{Conclusion}

In this paper, a new approach to generate artificial chaos by entangling two or multiple stable linear subsystems has been introduced. Meanwhile, review the linear stability analysis which accounts for the characterization, in the space of parameters, of the Lyapunov stability of the equilibria of the new system. It continues with the extension of the analysis to the first-order, codimension one Hopf bifurcation, based on the calculation of the first Lyapunov coefficient. At last, we controlled the system to any fixed point to eliminate the chaotic vibration by means of sliding mode method. And the numerical simulations were presented to confirm the effectiveness of the controller. Apparently there are more interesting problems about this chaotic system in terms of complexity, control, and synchronization, which deserve further investigation. Therefore, further research into the system is still important and insightful.

\section{Conflict of Interests}

The authors declare that there is no conflict of interests regarding the publication of this paper.

\section{Acknowledgments}

The authors gratefully acknowledge the support from the National Natural Science Foundation (nos. 11161027 and 61364001), the Key Project of Chinese Ministry of Education (no. 212180), and the Fundamental Research Funds for the Universities of Gansu Province (no. 620023) Government of China.

\section{References}

[1] E. N. Lorenz, "Deterministic nonperiodic flow," Journal of the Atmospheric Sciences, vol. 20, pp. 130-141, 1963.

[2] O. E. Rossler, "An equation for continuous chaos," Physics Letters A, vol. 57, no. 5, pp. 397-399, 1976.

[3] G. Chen and T. Ueta, "Yet another chaotic attractor," International Journal of Bifurcation and Chaos, vol. 9, no. 7, pp. 14651466, 1999.

[4] J. Lü and G. Chen, "A new chaotic attractor coined," International Journal of Bifurcation and Chaos in Applied Sciences and Engineering, vol. 12, no. 3, pp. 659-662, 2002.

[5] C. Liu, T. Liu, L. Liu, and K. Liu, "A new chaotic attractor," Chaos, Solitons and Fractals, vol. 22, no. 5, pp. 1031-1038, 2004.

[6] S. Celikovsky and G. Chen, "On the generalized Lorenz canonical form," Chaos, Solitons and Fractals, vol. 26, no. 5, pp. 12711276, 2005.

[7] Q. G. Yang, G. R. Chen, and K. F. Huang, "Chaotic attractors of the conjugate Lorenz-type system," International Journal of Bifurcation and Chaos, vol. 17, no. 11, pp. 3929-3949, 2007.

[8] G. van der Schrier and L. R. M. Maas, "The diffusionless Lorenz equations; Shil'nikov bifurcations and reduction to an explicit map," Physica D, vol. 141, no. 1-2, pp. 19-36, 2000.

[9] R. Shaw, "Strange attractors, chaotic behavior, and information flow," Zeitschrift für Naturforschung A, vol. 36, no. 1, pp. 80-112, 1981.

[10] Q. G. Yang and G. R. Chen, "A chaotic system with one saddle and two stable node-foci," International Journal of Bifurcation and Chaos, vol. 18, no. 5, pp. 1393-1414, 2008.

[11] G. Qi, G. Chen, S. Du, Z. Chen, and Z. Yuan, "Analysis of a new chaotic system," Physica A, vol. 352, no. 2-4, pp. 295-308, 2005.

[12] Q. Yang and Y. Chen, "Complex dynamics in the unified Lorenztype system," International Journal of Bifurcation and Chaos, vol. 24, no. 4, Article ID 1450055, 30 pages, 2014.

[13] D. Chen, P. Yang, X. Ma, and Z. Sun, "Chaos of hydro-turbine governing system and its control," Proceedings of the Chinese Society of Electrical Engineering, vol. 31, no. 14, pp. 113-120, 2011.

[14] D.-Y. Chen, Y.-X. Liu, X.-Y. Ma, and R.-F. Zhang, "Nochattering sliding mode control in a class of fractional-order chaotic systems," Chinese Physics B, vol. 20, no. 12, Article ID 120506, 9 pages, 2011.

[15] J.-H. Lee, P. E. Allaire, G. Tao, and X. Zhang, "Integral slidingmode control of a magnetically suspended balance beam: analysis, simulation, and experiment," IEEE/ASME Transactions on Mechatronics, vol. 6, no. 3, pp. 338-346, 2001.

[16] J. Wang, T. Lee, and Y. Juang, "New methods to design an integral variable structure controller," IEEE Transactions on Automatic Control, vol. 41, no. 1, pp. 140-143, 1996.

[17] D. Chen, T. Shen, and X. Ma, "Sliding mode control of chaotic vibrations of spinning disks with uncertain parameter under bounded disturbance," Acta Physica Sinica, vol. 60, no. 5, Article ID 050505, 2011.

[18] H. Zhang, X. Liu, X. Shen, and J. Liu, "Chaos entanglement: a new approach to generate chaos," International Journal of Bifurcation and Chaos in Applied Sciences and Engineering, vol. 23, no. 5, Article ID 1330014, 17 pages, 2013.

[19] A. Wolf, J. B. Swift, and H. L. a. Swinney, "Determining Lyapunov exponents from a time series," Physica D: Nonlinear Phenomena, vol. 16, no. 3, pp. 285-317, 1985.

[20] F. S. Dias, L. F. Mello, and J.-G. Zhang, "Nonlinear analysis in a Lorenz-like system," Nonlinear Analysis: Real World Applications, vol. 11, no. 5, pp. 3491-3500, 2010.

[21] Y. A. Kuznetsov, Elements of Applied Bifurcation Theory, Applied Mathematical Sciences, Springer, New York, NY, USA, 3rd edition, 2004. 


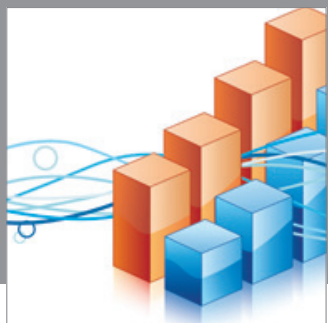

Advances in

Operations Research

mansans

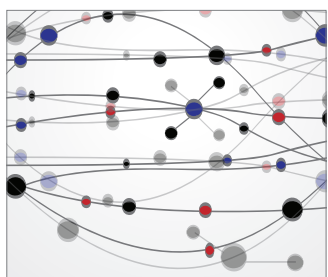

The Scientific World Journal
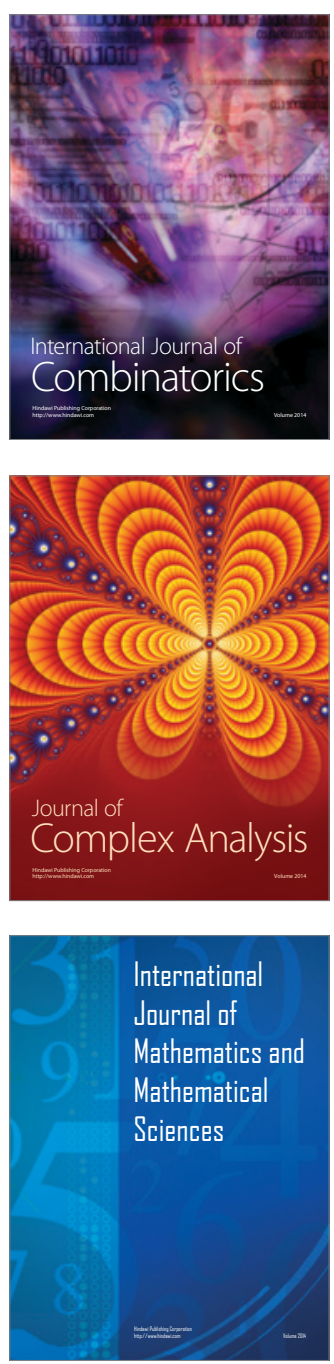
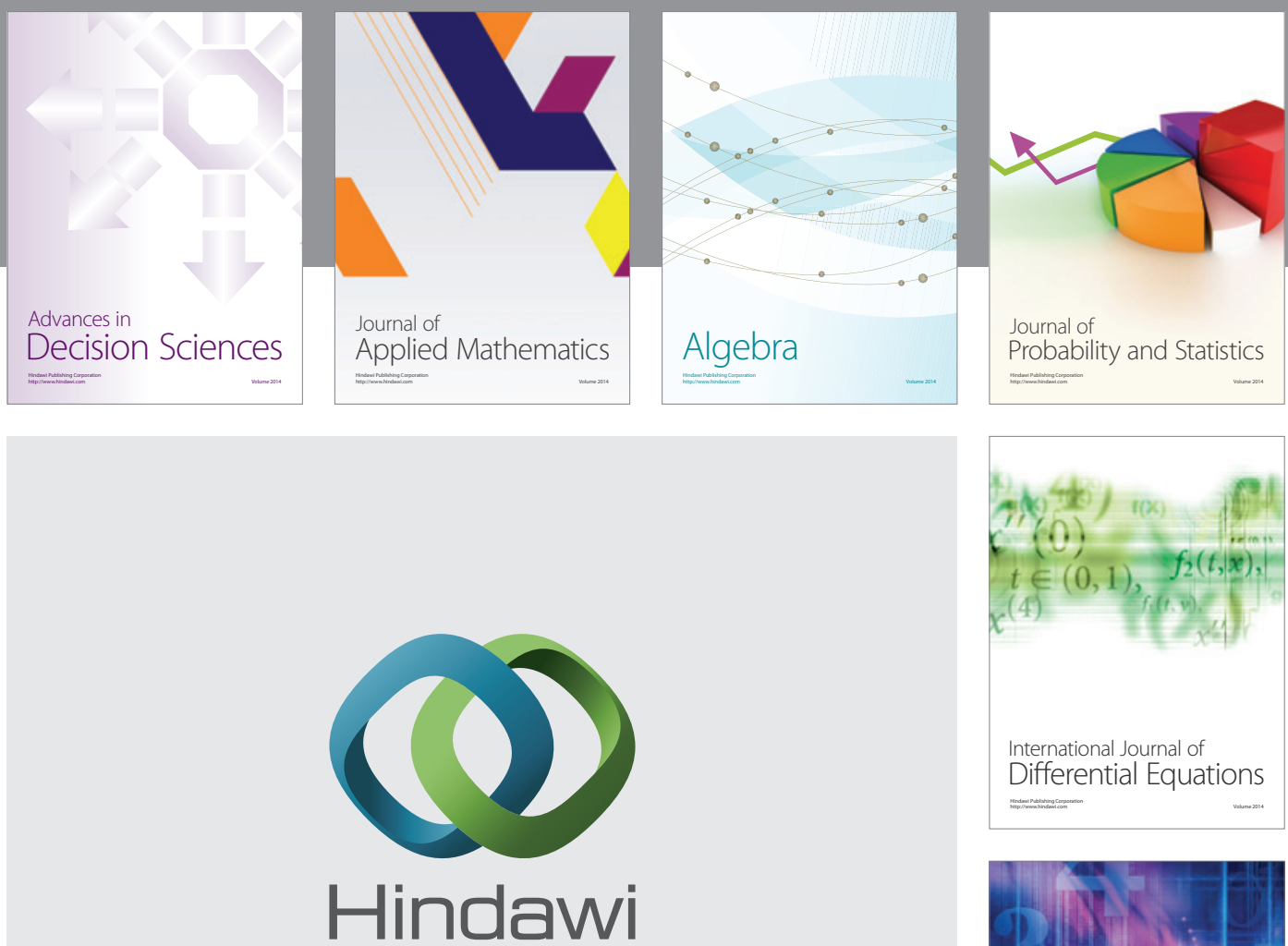

Submit your manuscripts at http://www.hindawi.com
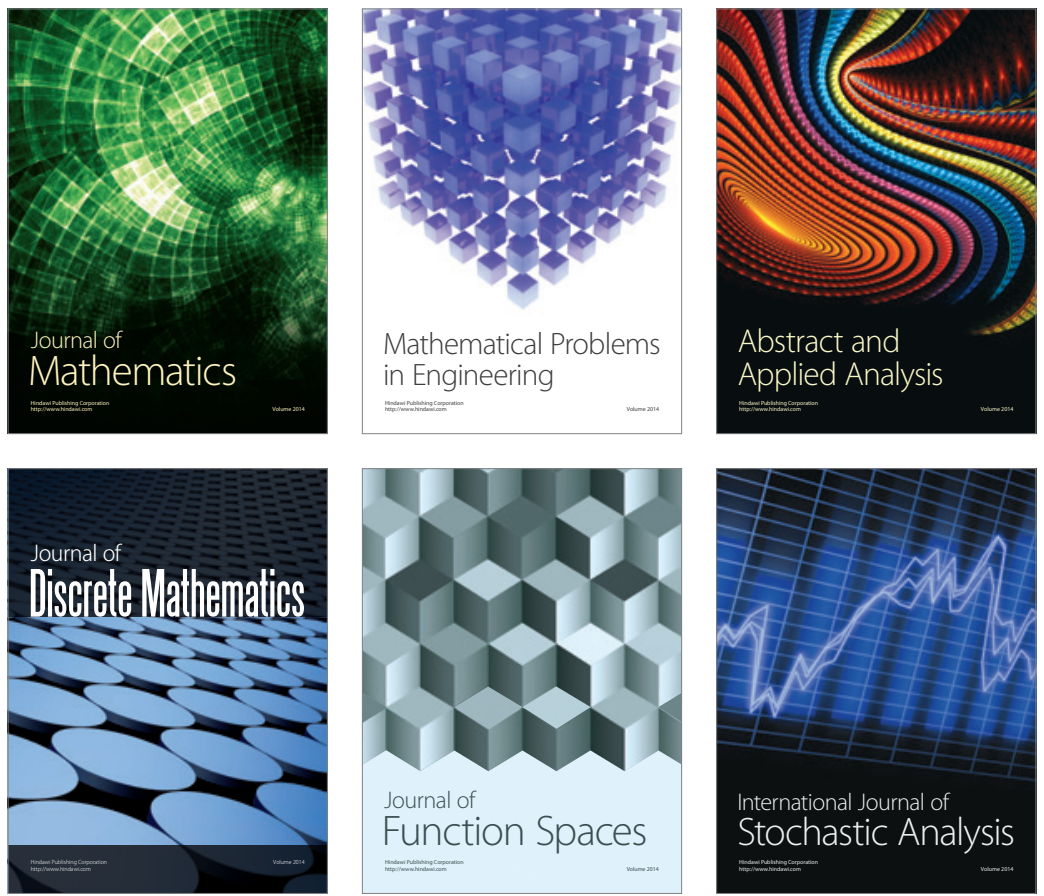

Journal of

Function Spaces

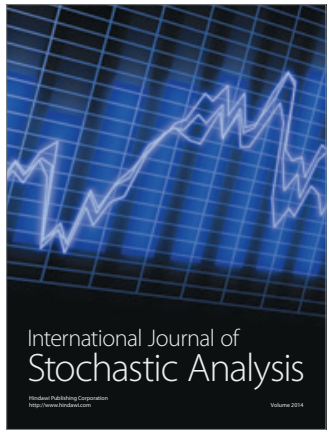

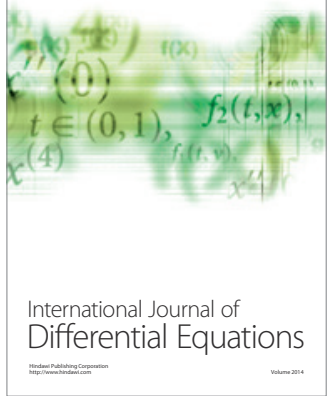
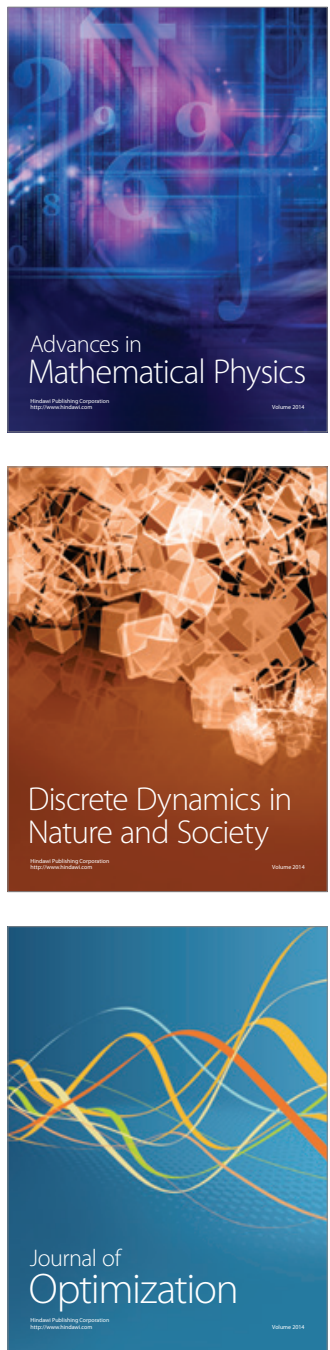